\title{
Experimental turbulence studies for gyro-kinetic code validation using advanced microwave diagnostics
}

U. $\operatorname{Stroth}^{1,2}$, A. Bañón Navarro ${ }^{1}$, G.D. Conway ${ }^{1}$, T. Görler ${ }^{1}$,

T. Happel ${ }^{1}$, P. Hennequin ${ }^{3}$, C. Lechte ${ }^{4}$, P. Manz ${ }^{2,1}$,

P. Simon ${ }^{1,4,5}$, A. Biancalani ${ }^{1}$, E. Blanco ${ }^{6}$, C. Bottereau $^{7}$,

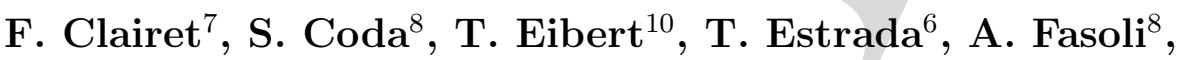

L. Guimarais ${ }^{9}$, Ö. Gürcan ${ }^{3}$, Z. Huang ${ }^{8}$, F. Jenko ${ }^{1}$,

W. Kasparek ${ }^{4}$, C. Koenen ${ }^{10}$, A. Krämer-Flecken ${ }^{11}$,

M.E. Manso ${ }^{9}$, A. Medvedeva $a^{1,2,7,5}$, D. Molina ${ }^{7}$, V. Nikolaeva ${ }^{9,1,2}$,

B. Plaum ${ }^{4}$, L. Porte $^{8}$, D. Prisiazhniuk ${ }^{11,1,2}$, T.Ribeiro ${ }^{1}$,

B.D. Scott ${ }^{1}$, U. Siart ${ }^{10}$, A. Storelli ${ }^{3}$, L. Vermare ${ }^{3}$, S. Wolf ${ }^{4}$ and the ASDEX Upgrade team

${ }^{1}$ Max-Planck-Institut für Plasmaphysik, Garching and Greifswald, Germany;

${ }^{2}$ Physik-Department E28, Technische Universität München, 85747 Garching,

Germany; ${ }^{3}$ École Polytechnique, CNRS UMR7648, Laboratoire de Physique des

Plasmas, F-91128, Palaiseau, France; ${ }^{4}$ Institut für Grenzflächenverfahrenstechnik und

Plasmatechnologie, Universität Stuttgart,70333 Stuttgart, Germany; ${ }^{5}$ Institut Jean

Lamour, Université de Lorraine, 54011 Nancy, France; ${ }^{6}$ Laboratorio Nacional de

Fusion, CIEMAT, Madrid, Spain; ${ }^{7}$ IRFM, CEA, F-13108 Saint Paul-Lez-Durance,

France; ${ }^{8}$ École Polytechnique Fédérale de Lausanne, CRPP, Switzerland; ${ }^{9}$ Instituto de Plasmas e Fusão Nuclear, Instituto Superior Técnico Universidade Técnica de Lisboa, 1049-001 Lisboa, Portugal; ${ }^{10}$ Lehrstuhl für Hochfrequenztechnik, Technische Universität, München, Germany; ${ }^{11}$ Institut für Energie- und Klimaforschung, Forschungszentrum Jülich, 52425 Jülich, Germany 
Abstract. For a comprehensive comparison with theoretical models and advanced numerical turbulence simulations, a large spectrum of fluctuation parameters were measured on the devices ASDEX Upgrade, TCV, and Tore-Supra. Radial profiles of scale-resolved turbulence levels in $\mathrm{H}$-mode discharges are measured and compared with GENE simulations in the transition range from ion-temperature-gradient to trappedelectron-mode turbulence. Correlation reflectometry is used to study the microscopic structure of turbulence and GAMs in discharges where poloidal flow damping was varied by means of variations of the shape of the poloidal plasma cross-section and collisionality. The aspects of turbulence spreading and non-local transport in response of local changes in density and temperature gradients are studied during phases with ECRH power modulation by means of radial correlation measurements and an ultrafast reflectometer. Full-wave codes and synthetic diagnostics are applied for the interpretation of the data.

\section{Introduction and strategy}

The investigation of the physical processes underlying turbulent transport remains an outstanding topic in fusion research with fascinating scientific aspects and great importance for enabling predictive transport modeling for future fusion devices. Progress in this field needs a coherent experimental and theoretical effort. Due to increasing computer power and the development of simulation codes based on gyrokinetic or gyro-fluid theory, detailed predictions of the microscopic properties of the turbulence have become available. On the basis of measured kinetic profiles for ion and electron temperatures and densities, turbulence simulation codes are nowadays capable of reproducing the experimental particle and power fluxes in selected plasma regimes. In order to ensure that these promising results are obtained on the basis of the correct physical models, detailed comparisons of numerical simulations with experimental data have to be carried out on a microscopic level, i.e. by comparing the characteristics of the fluctuations in the various plasma parameters, which is usually referred to as code validation. 
Due to the limited experimental accessibility of the relevant parameters, such comparisons are, however, not straight forward to be executed. Microwave-based diagnostics represent one of the few experimental tools to access plasma parameter fluctuations with the required spatio-temporal resolution. Conventional and Doppler reflectometry can provide scale resolved information on density fluctuations while the measurement of the electron-cyclotron emission (ECE) can be used to obtain data on electron temperature fluctuations. A common difficulty of microwave-based diagnostics is that the measured signals are produced through a not always trivial interaction of the wave with the turbulent plasma. Hence, often such diagnostics yield only an indirect access to the fluctuating parameters. When non-linear processes start to affect the interaction between microwave and fluctuations, full-wave codes play a major role in extracting quantitative information from the measured signals. Therefore our strategy to a detailed experiment-theory comparison relies not only on (i) high-level simulation codes and (ii) advanced microwave diagnostics, but also on (iii) full-wave simulations of the microwave beam to link the measured signal to the turbulent plasma background.

While results from simulation codes comprise rather complete information on the microscopic structure of the turbulence, such as two-dimensional fluctuation profiles of all relevant parameters and the important cross-phases between different parameter fluctuations, as well as the dynamics of zonal flows regulating the turbulence, the experiment in general only provides data on the spatio-temporal structure of the density and temperature fluctuations and on locally measured flows. Therefore an important element of a viable strategy is also the identification of meaningful quantities for an experiment-theory comparison.

The present contribution reports on an extensive international effort undertaken on the devices ASDEX Upgrade, TCV, and Tore-Supra to measure simultaneously a large spectrum of fluctuation parameters in order to achieve a comprehensive comparison with theoretical models and advanced numerical turbulence simulations. An overview is given of the status of the research project including first results. The project is 
also developing advanced hardware solutions for reflectometry on future devices such as ITER and DEMO. In particular phased array antennas are developed, which do not rely on moving parts inside the vacuum vessel. The phase delay is created either through a serial corkscrew feed [1] or via a novel phase shifter with piezo actuators and a broadband coupling structure [2]. Both systems have been systematically simulated, optimized, and finally prototyped and laboratory tested. A first version has been installation on AUG. Details on the hardware developments will be published elsewhere.

The article starts with a brief summary of the available diagnostics and then reports on first results on the characteristics of density fluctuations obtained in the plasma core at the transition from ITG to TEM turbulence, on the structure of density fluctuations, on the properties of flows in the plasma edge and on a first search for non-local features in transport.

\section{Microwave diagnostics and full-wave simulations}

The experimental work primarily relies on a suite of reflectometers which, at present, are mostly installed on the ASDEX Upgrade (AUG) tokamak but which have been or will also be used on the tokamaks TCV and Tore Supra. The diagnostics are used to obtain a comprehensive picture of the turbulent fluctuations in different confinement regimes from the plasma core to the edge.

Four Doppler reflectometers covering the frequency range $50-110 \mathrm{GHz}$ in $\mathrm{O}-$ and X-mode polarizations are installed using both fixed tilt antennas and an optimized scanning mirror for measurements of perpendicular wavenumber density fluctuation spectra, with a radial coverage from the plasma SOL (scrape-off layer) to the pedestal top or, at moderate densities, up to the plasma center $[3,4,5,6]$. From the Doppler shift in the frequency spectra, the perpendicular turbulence propagation velocity can be reliably derived to be used for the study of zonal flows and geodesic acoustic modes (GAMs). Cross-correlation analyses between different channels are employed to investigate turbulence spreading, the shear structure of flows, as well as flow turbulence 

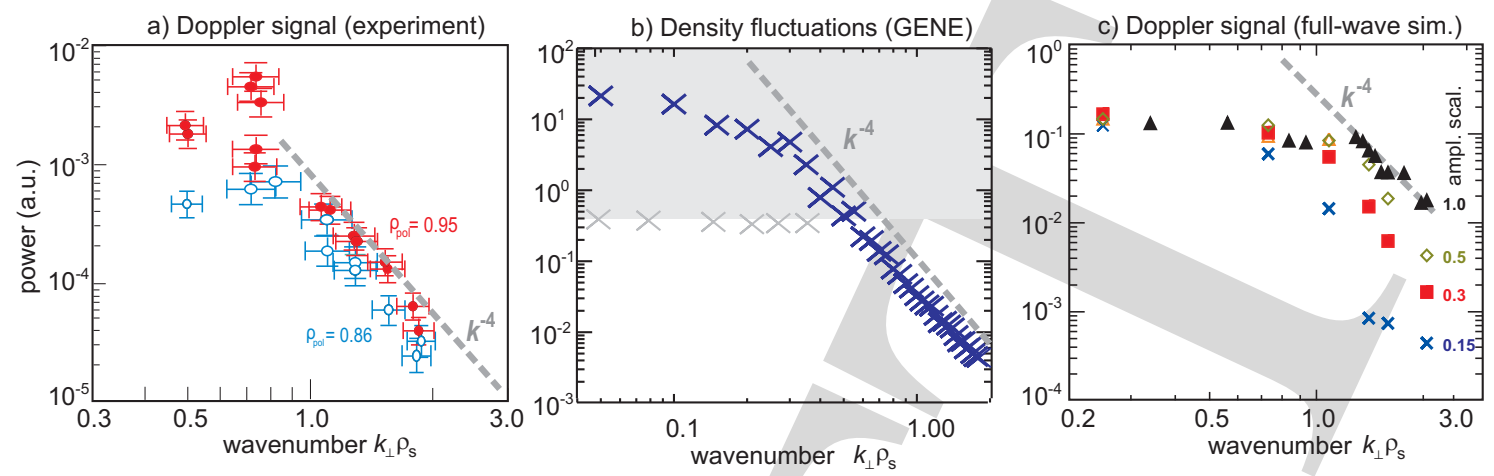

Figure 1. Comparison of wavenumber spectra from Doppler reflectometry on AUG measured at two normalized radii (a), from nonlinear GENE simulations in fluxtube geometry (b), and from the IPF-FD3D full-wave code (c) based on the density fluctuations from GENE where the amplitude was scaled as indicated in (c). The dashed grey lines are given as a reference for the slope of the spectra and the grey area and crosses in (b) indicate the effect of non-linear saturation (adapted from Ref. [11]).

interaction.

A V/W-band ultra-fast swept X-mode reflectometer measures up to 100,000 density profiles along a horizontal line of sight across the plasma center with $2 \mu$ s resolution [7]. The diagnostic is used to study the radial structure of turbulence as well as turbulence spreading in response to local changes in the background profiles as they can appear during L-H transitions or changes in electron-cyclotron heating power.

A multi-point correlation reflectometer operating in the range $26-60 \mathrm{GHz}$ with one emitting and four receiving antennas provides information on the radial and poloidal correlation lengths, the decorrelation times, and, through cross-correlation analyses, the turbulent propagation velocity of density fluctuations [8]. It is an ideal tool to study the two-dimensional structure of the turbulence with the potential to derive the safety-factor profile [9]. An important global aspect of turbulence is the poloidal asymmetry of the fluctuations which is addressed with two multi-channel O-mode reflectometer systems installed on the low and high-field sides of AUG [10].

For all applications, validations of the diagnostics with full-wave codes are envisaged. Figure 1 shall serve as an illustration of the effort a thorough comparison of experimental and simulated data on density fluctuations can take. Graph (a) depicts 
perpendicular wavenumber $\left(k_{\perp}\right)$ spectra measured at two radial position in L-mode plasmas on AUG using the W-band reflectometer with a steerable tilt antenna in X-mode polarization $[12,13]$. Experimental background plasma parameters at a normalized radius of $\rho_{\text {pol }} \approx 0.85$ were used as input to the GENE code [14] to carry out gyrokinetic turbulence simulations in flux-tube geometry resulting in the spectrum shown in graph (b). Both spectra have a Kolmogorov-like shape with a flat region at low $k_{\perp}$ followed by a power law decay at higher $k_{\perp}$. Characteristic parameters for a comparison are the location in $k_{\perp}$ of the knee joining the two regimes and the spectral index of the decay. Although the slopes in the experimental and simulated spectrum are similar, the transition from a flat "injection range" to the slanted "inertial range" appears in the simulation at smaller values $\left(k_{\perp} \rho_{s} \approx 0.3\right)$ than in the experiment $\left(k_{\perp} \rho_{s} \approx 0.8\right)$.

The two spectra can be reconciled through full-wave simulations of the microwave beam on the background of numerical turbulence data generated with the GENE code for the actual plasma discharge. Figure 1c depicts such $k_{\perp}$ spectra calculated with the IPF-FD3D full-wave code [15]. The different symbols refer to results obtained from density fluctuation data where the amplitude of the used turbulent field was rescaled by factors between 0.15 to 1.0 , as indicated on the right of the figure [15]. It is found that above a critical fluctuation amplitude the intensity of the received diffracted microwave beam is no longer proportional to the amplitude of the fluctuations at the probed wavenumber $[16,15]$. This leads to a deformation of the measured intensity spectrum with respect to the density fluctuation spectrum. For artificially small density fluctuation amplitudes, the knee in the synthetic spectrum appears at lower $k_{\perp}$ values, close to the one in the density fluctuation spectrum from GENE simulations, and the slope is somewhat steeper. Increasing the fluctuation strength to the actual level from the GENE simulations leads to a non-linear saturation of the power measured in the synthetic reflectometer. At high $k_{\perp}$, the calculated reflectometer signal is still in the linear phase and thus can increase with the fluctuation amplitude. This significantly alters the shape of the spectrum, shifting the knee position to higher wavenumbers, and 
making the slope in the inertial range somewhat more shallow. Hence, the observed experimental slope is quite similar to the slope from the reflectometer simulation at the appropriate fluctuation level, which means that, in this first study, the turbulent spectrum from the GENE simulations is consistent with the spectrum one would expect to measure experimentally [11]. In future studies relying on data with higher quality obtained with the new reflectometers, also the slope of the spectrum for $k_{\perp} \rho_{s}<1$ will be included in a more rigorous comparison with the simulations.

That non-linear effects can affect the signals from Doppler reflectometry has been shown analytically and a critical parameter has been derived above which non-linear effects have to be considered [17]. This Gusakov parameter obtained was confirmed to be relevant for the onset of non-linear behavior for O-mode reflectometry by 2D full-wave simulations in slab geometry [16]. Although the mechanism leading to the saturation discussed above might be similar, a quantitative comparison of the results from the present study with the Gusakov parameter is not appropriate since the analytical model was derived for O-mode polarisation in slab geometry for a linear background density profile while simulations and experiments reported here were done for X-Mode polarisation and with measured background profiles and realistic geometry.

Previous measurements of wavenumber spectra with a Doppler reflectometer on Tore Supra were carried out in O-mode polarisation [18]. The spectra exhibit two slopes; instead of a flat region at small wavenumbers, the spectrum decays with a spectral index of -3 for $k_{\perp} \rho_{S}<1$. The knee is located at about $k_{\perp} \rho_{S} \approx 1$ and for higher wavenumbers, a rather steep decay with a spectral index of the order -6 is observed. The measured spectra agree reasonably well with gyrokinetic simulations combined with a simple synthetic diagnostic [19] and with an analytical model [20]. The fact that these spectra obtained by Doppler backscattering are consistent with earlier results obtained by laser scattering [21], where non-linear saturation effects are excluded to play a role, might indicate that $\mathrm{O}$-mode reflectometry suffers less from non-linear saturation. But then it remains to be understood, why the shape of the O-mode spectra from Tore Supra 
is different from the one from the GENE simulation in Fig. 1b which represents the expected density fluctuation spectrum in an AUG L-mode discharge. Is the difference in the spectral shape attributed to the fact that the plasma parameters are different, or is the diffraction process in O-mode polarisation influenced by non-linear effects, too? The latter point will be addressed in the near future by means of full-wave simulations. It should also be mentioned that the importance of non-linear effects at low wavenumber can be reduced if large scale fluctuations are suppressed by a strong background flow shear as reported for the core of H-mode discharges in the DIII-D tokamak [22].

Synthetic diagnostic and comparisons between turbulence measurements and simulations have already been carried out previously (e.g. Refs. $[23,24,25,26]$ ). To our knowledge this is, however, the first time that the complete chain from measured wavenumber spectra through a full-wave code simulating Doppler reflectometry in realistic geometry to gyrokinetic simulations has been implemented and applied. Synthetic diagnostics often "just" involve appropriate mappings, volume integrations, and filters.

For the Doppler data presented in the next section, full-wave analyses have not

yet been carried out. However, since the measurement were taken in H-mode plasmas and further in the plasma core, where the fluctuation amplitudes are smaller, non-linear effects should be of less importance. Furthermore, flow measurements are in general not affected by the non-linear wave-plasma interaction [16].

\section{ITG turbulence in the plasma core}

The plasma core at parameters where a transition from ion-temperature-gradient (ITG) to trapped-electron-mode (TEM) driven turbulence occurs is just the right area for a detailed theory-experiment comparison. Experimental observations on momentum and particle transport, such as density peaking or, to some extent, finite intrinsic rotation velocities in the absence of an appropriate particle source or external momentum input, respectively, [27, 28] could be successfully described with transport coefficients derived 
from linear calculations with the gyro-kinetic model GS2 [29, 30], pointing to changes in the residual stress through a density gradient dependence in the ITG-TEM transitional region as a main cause. Theoretical studies have shown [31] that the transition from the ITG to the TEM regime enhances the turbulent particle pinch and thus can change the density peaking and the rotation profile flattens which is qualitatively consistent with an experimental analysis [27]. More details on particle transport in the core of plasmas at the ITG-TEM transition can be found in Ref. [32].

As a basis for experiment-theory comparisons of the turbulent structure, H-mode discharges at parameters relevant for the above observations were carried out in AUG where up to $1.8 \mathrm{MW}$ of ECRH power was optionally deposited at different radial locations to modify the local electron temperature gradient. This affects the TEM stability and the turbulence regime is expected to shift from the ITG towards the TEM type. One should, however, not expect that the turbulent state is purely ITG or TEMlike, in reality it will be rather a mixture of both types of modes and also stable modes can be exited through non-linear interaction. But these effects are included in the GENE code which is used for comparison [33].

Radial profiles and wavenumber spectra of the perpendicular turbulence propagation velocity and the density fluctuation amplitudes have been measured with the W-band Doppler reflectometer and comparisons with linear and non-linear flux-tube simulations from the gyro-kinetic turbulence code GENE [14] have been carried out $[34,35]$. For linear simulations, the resolution of the numerical grid was $\{31 \times 32 \times 48 \times 16\}$ in $\left\{x, z, v_{\|}, \mu\right\}$ coordinates, where $\mu$ is the magnetic moment, and in non-linear simulations it was $\{256 \times 128 \times 32 \times 48 \times 16\}$ in the coordinates $\left\{x, y, z, v_{\|}, \mu\right\}$. The non-linear simulation box size was $\left\{125 \rho_{s} ; 125 \rho_{s} ; 2 \pi ;[-3,3] v_{t h} ;[0,9] T / B\right\}$ where $v_{t h}$ is the thermal velocity. A realistic electron-ion mass ratio was used and the shearing rate was small compared to the maximum growth rate of the dominant instability. More numerical details of the simulations can be found in Refs. [35, 34].

In order to match the experimental electron and ion heat fluxes in the GENE 

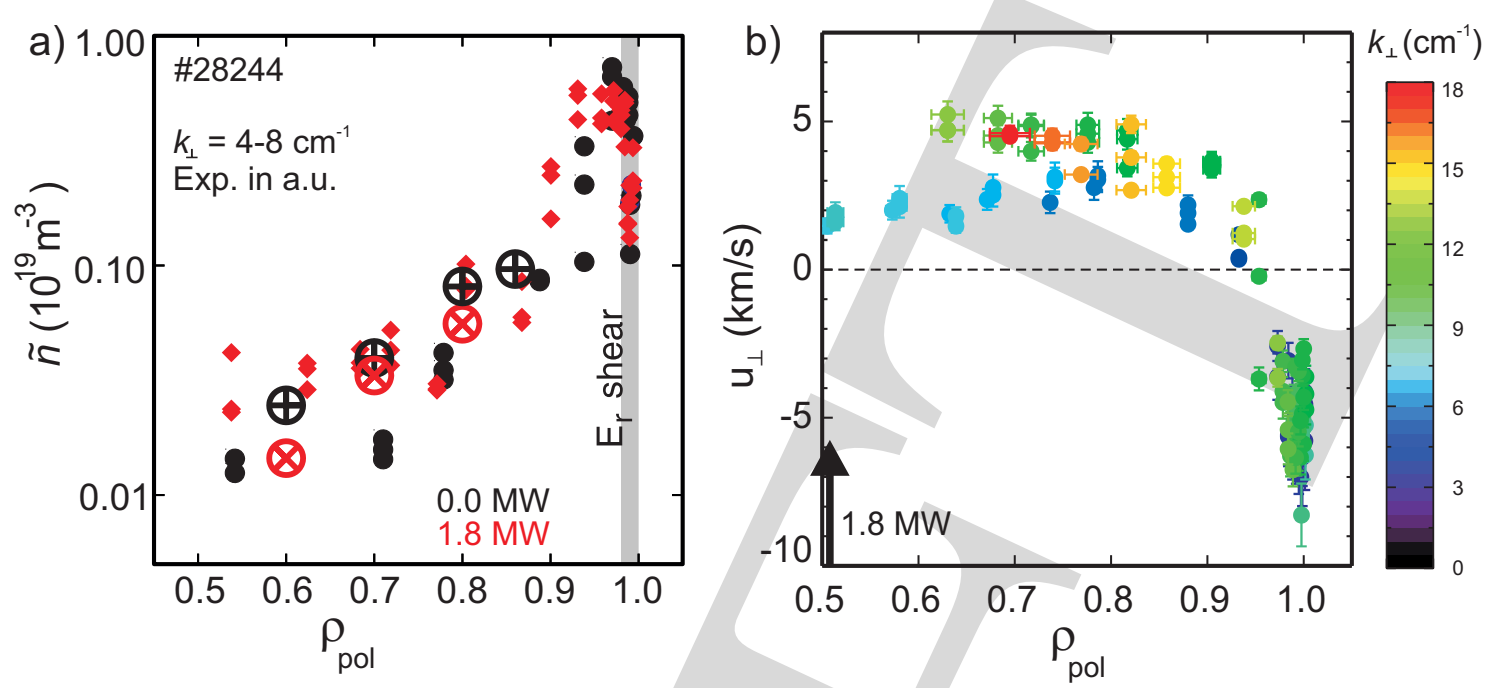

Figure 2. a) Comparison of radial profiles of density fluctuation amplitudes from Doppler reflectometry multiplied by a constant factor and flux-matched GENE simulations (big circles). b) Radial profiles of the perpendicular propagation velocity of density fluctuations measured at different values of $k_{\perp}$ (color coded). (adapted from $[34])$.

simulations, the ion temperature gradient had to be reduced within the experimental uncertainty by up to $20 \%$. Under this restriction, non-linear GENE simulations succeed in reproducing simultaneously both the experimental power fluxes and the radial increase in the turbulence level by a factor of five from the core to the edge, as depicted in Fig. 2a. Since the Doppler measurements do not yield absolute fluctuation amplitudes, the data have been renormalized by a constant factor. The suppression of turbulence visible close to the plasma edge is attributed to a strong shear layer in the background $E \times B$ flow, which is generally observed in H-mode. The simulations were carried out in the radial region where the strongest effect due to a transition from ITG to TEM turbulence could be expected. The region $\rho_{\text {pol }}>0.9$ was also excluded since strong shear flows start to play a role which might introduce additional effects mixing with the ones under observation. In all scenarios and at all radial positions studied, turbulence is predicted by linear simulations to be in the ITG regime, but the TEM stability is found to decrease when $1.8 \mathrm{MW}$ of ECRH is added at about mid plasma radius. In 
the latter case the experimental fluctuation amplitudes increase by about a factor of two. This trend is not yet reproduced by the gyrokinetic simulations, where the density fluctuation amplitudes weakly decrease.

The wavenumber dependent turbulence propagation velocities are less affected by the details of wave diffraction, as e.g. shown by means of full-wave simulations [16], and thus can be used directly for a comparison with theory. Since the background flow is the same for all wavenumber components, such measurements can be used to extract the incremental phase velocity and the turbulence dispersion relation, both being indicative for the type of turbulence. In discharges without additional ECRH, all spectral components propagate at the same speed of about $5 \mathrm{~km} / \mathrm{s}$ in the core, indicating that the change of phase velocity with $k_{\perp}$ is small compared to the background flow [34]. With increasing ECRH power up to 1.8 MW deposited at mid radius, as shown in Fig. 2b, the propagation velocity of small and intermediate-scale fluctuations remains unaffected while large-scale structures slow down. This corresponds to an increased phase velocity of the large scales towards the electron-diamagnetic direction, an observation consistent with an increased influence of TEM turbulence which also travels into this direction. Also the increase in the turbulence level shown in Fig. 2a is mainly attributed to the larger scales. Both effects are consistent with a transition from a clearly ITG dominated plasma towards the TEM regime, either driven by a steeper electron temperature gradient or by an increased density gradient [34]. The observed change in propagation velocity of $3 \mathrm{~km} / \mathrm{s}$ is, however, about a factor of 10 larger than expected from linear gyrokinetic simulations.

For the experimental parameters investigated, linear gyrokinetic simulations show that the ITG is the fastest growing mode, and analyses of non-linear simulations yield positive phase velocities, which correspond in the GENE reference frame to ITG modes. The fact that ITG is dominant even with strong ECRH can be explained taking into account that the measurements were taken in the outer half of the minor plasma radius, where $T_{e} / T_{i} \approx 1$ and $\approx 1.4$ without and with $1.8 \mathrm{MW}$ of additional ECRH, respectively. 
Both $T_{e} / T_{i}$ and the density scale length $R / L_{n}$ increase substantially with increased electron heating, such that the ITG mode becomes more unstable. Furthermore, trapped electrons are not exclusively related to the TEM instability. Instead, they have been shown to play a major role in the ITG instability, too $[36,37]$.

In the same context fall previous experiments on Tore Supra. The wavenumber dependence of the turbulent propagation velocity was measured with Doppler reflectometry in experiments where the normalized collisionality $\nu^{*}$ was varied by a factor four while other dimensionless parameters where kept constant [18]. At a given radius a clear modification of the turbulent propagation velocity was observed between the two collisionality cases. In the high collisionality discharge, where ITG turbulence should be dominant, the perpendicular velocity, which due to the background flow points into the electron-diamagnetic direction, is found to decrease by almost a factor 2 in the entire range from small to large wavenumbers while at low collisionality, in a mixed ITG/TEM regime, the velocity is larger and remains almost constant over the entire wavenumber range probed $\left(k \rho_{i}=0.5-1.4\right)$ [18]. The strongest change in velocity of the order of $1 \mathrm{~km} / \mathrm{s}$ is again by about a factor of ten larger than predicted by gyrokinetic simulations [38].

On TCV, studies of core turbulence have focused on the dependence of the fluctuation characteristics on plasma triangularity, in a TEM-dominated regime. Global confinement improves substantially in going from positive to negative triangularity [39], an effect that appears, however, to be governed entirely by changes in normalized gradients at the edge [40], consistent with the poor penetration of triangularity into the core; accordingly, flux-tube gyrokinetic simulations saw the effect of triangularity diminish and eventually vanish in going from the edge to the core [41]. The amplitude of broadband density and temperature fluctuations, measured by phase contrast imaging (PCI) and correlation ECE, respectively, is however higher at positive triangularity well into the core [42]. These somewhat conflicting observations remain to be reconciled through global nonlinear gyrokinetic simulations. An increase in the turbulence 
level with decreasing effective collisionality was also seen by PCI, consistent with a concomitant increase in transport [42].

\section{Geodesic acoustic modes}

Zonal flows and geodesic acoustic modes (GAMs) are an inherent element of turbulence in magnetically confined plasmas. They are known to play an important role in the regulation of the turbulence level and thus must be part of a comprehensive comparison between theory and experiment. Radial correlation Doppler reflectometry is an ideal tool to measure the spatio-temporal GAM structure and its dependence on plasma shape and collisionality.

In discharges on AUG, poloidal flow damping was varied through changes in the poloidal plasma cross-section and the isotope mass. The order of magnitude of the GAM frequency is generally well described by the ratio of the sound speed and the major plasma radius, $c_{s} / R$. However, the plasma shape also influences the GAM frequency which was parameterised by an empirical scaling law depending on the plasma elongation $\kappa[13]$. In a recent experiment, a systematic variation was carried out from a circular limiter to an elongated divertor L-mode plasma. A database containing the local plasma parameters and the GAM properties of this configuration scans was analyzed recently and the results are presented in Ref. [43]. The empirical parametrization still describes the overall trend in the GAM frequency but systematic deviations are now observed when comparing edge to core GAMs and divertor to limiter ones. More comprehensive analytical models, as the one by Gao et al. [44], overestimate the GAM frequency by about $30 \%$; the systematic scatter in the data is, however, reduced [45]. In the configuration scan on AUG, circular plasmas exhibit the strongest GAM activity which is in qualitative agreement with the reduced damping when compared with elongated plasmas. Also on the Tore Supra it is observed that advanced analytical models predict higher frequencies than actually seen in the experiments [46]. In MAST, on the other hand, the aspect ratio dependence of the GAM frequency is well described with a global 

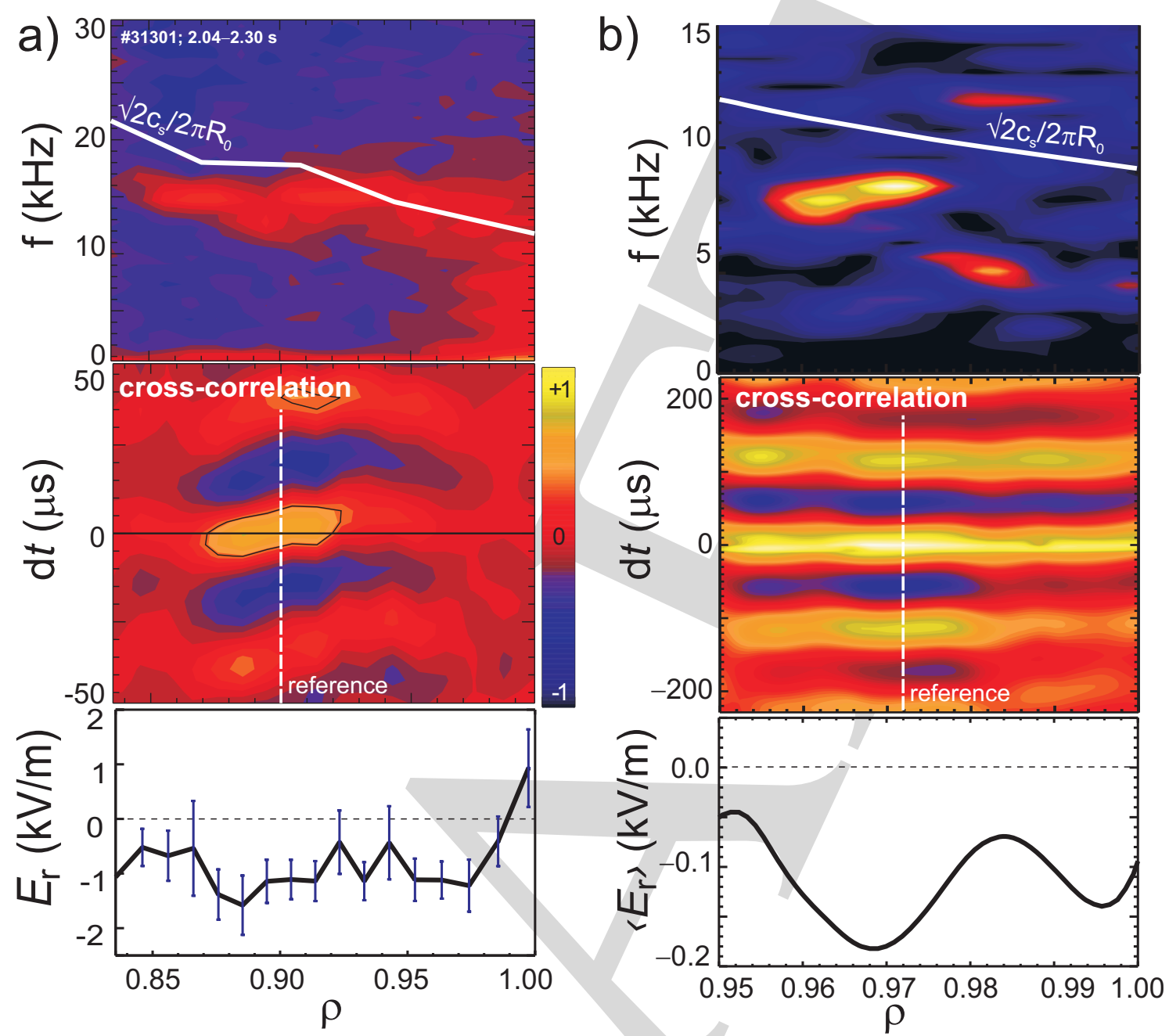

Figure 3. Spatio-temporal GAM structure for different plasma elongations $\kappa$ from Doppler-correlation reflectometry (a) and from GEMR gyrofluid simulations (b).

two-fluid code CENTORI [47].

A more meaningful comparison can be achieved by comparing spatio-temporal data from Doppler measurements and from turbulence codes. For an AUG discharge with a limited circular L-mode plasma, a simulation with the gyrofluid code GEMR [48] was used as a reference. The code has been developed to simulate turbulence at the plasma edge, where the GAM activity is strongest.

Figure 3a depicts the experimental results of a cross-correlation analysis between flows measured with one fixed-frequency channel and one where the probing frequency and thus the radial position was varied. As shown in the upper graph, the GAM develops with a constant and phase locked frequency over a wide radial region and does 
not follow the local $c_{s} / R$ estimated shown in the figure. This is a signature of a global GAM mode in contrast to continuum GAMs, where the frequency follows the local variation of the sound speed. The graph below depicts the cross-correlation function between the two channels. One finds structures with a finite correlation time which are non-monotonically tilted without indicating a clear radial propagation of the GAM mode. It seems rather to be locked in the radial electric field $\left(E_{r}\right)$ well shown in the lower graph as deduced from the Doppler spectra. The $E_{r}$ well in L-mode discharges was also studied by charge-exchange spectroscopy on AUG [49].

A qualitatively similar behavior is found in the GEMR simulations represented in Fig. 3b which were obtained, as in the experiment, for a circular limiter L-mode plasma where the local plasma parameters differed from the experimental ones as it can be seen from the $c_{s} / R$ estimates plotted in upper figures. As in the experiment, also in the GEMR simulations the dominant GAM mode is excited at one frequency, hence there is no reason to invoke a GAM dispersion relation. The GAM structure in the simulation is not tilted and the envelope stays, as in the experiment, at a fixed position in an $E_{r}$ well. Thus the interpretation of a tilt as a phase velocity would only make sense if the GAM is continuously excited on one side and damped on the other side, a questionable picture. A possible reason for the GAM being observed in the region of the $E_{r}$ well could be that a background shear flow tilts the turbulent eddies and thus activate Reynolds stress drive which in turn increase zonal flow drive with a coupling into the GAM.

Also on TCV the conditions were investigated for a GAM to appear either in the global or in the continuum form. In earlier studies, simulations with the global gyrokinetic PIC code ORB5 described fairly well data from the PCI diagnostic where the GAM frequency assumed constant values over a wide radial area, i.e. the outer $30 \%$ of the plasma cross section [50]. Later on, however, a transition from eigenmode to continuum GAMs was observed as the safety factor was systematically increased [42]. Transitions between global and continuum GAMs have also been observed on DIII-D [51] and MAST [47] while on JFT-2M a constant GAM frequency over a few centimetres 
was measured with a heavy ion beam probe [52].

As e.g. on JFT-2M [52], also on TCV a statistically significant bicoherence is measured between the GAM and the broadband turbulence, indicative of nonlinear interaction. In a density ramp, a strong increase in the turbulence level is observed, whereas the GAM level remains approximately constant, suggesting that the increased nonlinear GAM drive may be compensated by the increase in collisional damping [42].

On Tore Supra a Doppler reflectometer was used for GAM studies. The GAM frequency varies over a wider radial range (typically from $\rho=0.8-0.98$ ) and roughly follows the local sound speed. But this continuous trend is also interrupted by smaller frequency plateaus of the order $\Delta \rho=0.05$ which suggest the existence of eigenmodes [46]. Furthermore, the dependence of the GAM properties on collisionality has been investigated [53] and for a high collisionality discharge the experimental observations have been compared with a dedicated simulation from the gyrokinetic code GYSELA [54]. Here the GAM frequency appears to be overestimated by the simulation and, for both collisionalities, also by theoretical models. The disagreement appears stronger at low collisionality. The GAM activity is found to be stronger in the low collisionality case which is consistent with the fact that in areas of interest the dominant damping is through ion-ion collisions. GENE linear and non-linear simulations predict a stronger GAM drive in the low collisionality case, too [46]. In addition, a comparison on Tore Supra of the GAM dynamics exhibits similar probability density function of the GAM intensity and burst autocorrelation times in the simulation and the experiment [46].

Although linear analytical models capture the major trends of the experimental data, the GAM frequency dependence on plasma shape, the transition from global to continuum GAM modes and related to that the detailed dependence of the GAM frequency on local plasma parameters are not yet well understood. The nonlinear modification of the GAM frequency due to interaction with drift waves has been recently addressed by means of gyrokinetic analytical theory $[55,56]$. A more detailed comparison with gyrokinetic numerical simulation and analytical theory is in progress. 


\section{Spatial structure of turbulence}

The isotope effect, which refers to longer energy confinement times in deuterium as compared to hydrogen plasmas, remains an unsolved puzzle in fusion research. Since turbulent structures are expected to increase with isotope mass $m_{i}$ proportional to the drift scale $\rho_{s} \sim \sqrt{m_{i}}$, higher transport coefficients are expected rather for deuterium plasmas. Theoretically, this effect could be overcompensated by a stronger zonal flow activity in deuterium, where the collisional damping of zonal flows is lower due to the

lower ion-ion collision frequency $\nu_{i i} \sim 1 / \sqrt{m_{i}}$. For example, on TEXTOR, a tokamak with a circular limited plasma, an increase of the zonal flow activity from hydrogen to deuterium was observed [57]. On AUG, comparative discharges have been carried out in hydrogen and deuterium and the radial correlation lengths were measured by means of radial correlation Doppler reflectometry. As theoretically expected, an increase in the characteristic size of the turbulent structures for deuterium plasmas was observed in the plasma core [58]. This of course would rather lead to a reduction of the confinement time from hydrogen to deuterium in contrast to the observed increase. But the main contribution to the kinetic energy content of the plasma is generated at the plasma edge and the parameters at the pedestal top have a strong influence on the profiles in the core. Hence the scaling in the pedestal is more relevant for the global confinement time than that in the core. And indeed is the scaling of the size of the turbulent structures with the isotope mass in the plasma edge weaker or even absent [58]; also in the scrapeoff layer a rather weak $\rho_{s}$ dependence was measured with a lithium-beam diagnostic on AUG [59]. This still does not explain the isotope effect of the confinement time, but in addition to the turbulent scales the cross-phases between density and temperature fluctuations on the one-hand side and the potential fluctuations on the other determine the transport level, too. The analyses of the zonal flow and GAM activity as well as the discussion of their impact on the $\rho_{s}$ dependence of the fluctuation amplitudes is ongoing.

Apart from studies of the isotope effect, poloidal asymmetries in the fluctuations 
were investigated. Turbulence simulations exhibit strong poloidal asymmetries in the fluctuation amplitudes which are consistent with the location of the strongest turbulence drive in the bad-curvature region of the low-field side of the plasma. This asymmetry and the question about the strength of the communication between high and low-field sides was experimentally addressed with frequency hopping reflectometers operated at fixed frequencies to probe different radial positions located at the inboard and outboard sides of the vacuum vessel. In L-mode discharges in different configurations, including upper and lower single null as well as double null shapes, the poloidal asymmetry of density fluctuations in the edge and the SOL of the plasma were measured. As expected, strong poloidal asymmetries in the density fluctuation amplitudes were found with the strongest activity in the bad curvature region both in the edge and the SOL. In all cases, the asymmetry is strongest outside of the separatrix. The lowest fluctuation levels were observed in the good curvature region of the SOL in the double-null configuration, where it is decoupled from the bad-curvature region by two X-points. The difference in the fluctuation amplitudes inside and outside of the separatrix were strongest in the double-null situation, as it was also observed in three-dimensional electromagnetic gyrofluid computations with the GEM code [60].

\section{Conclusions}

The experimental validation of the physical models implemented in turbulence simulation codes for fusion plasmas has to be carried out on the microscopic basis of plasma parameter fluctuations. Microwave-based diagnostics, and reflectometry in particular, can provide relevant data for such a task. In many cases a direct comparison can, however, only be done by means of synthetic diagnostics based on full-wave simulations of the microwave diffraction in a simulated turbulent plasma. In the case of wavenumber spectra from Doppler reflectometry such a procedure has improved the agreement between experimental spectra from AUG and those from GENE simulations.

First comparisons of density fluctuation data from experiment and gyrokinetic 
simulations obtained in a transitional regime from ITG to TEM turbulence are encouraging. The radial increase in the fluctuation amplitudes and the heat transport are simultaneously quantitatively reproduced. Other details of the comparison, such as the dependence on temperature gradient or collisionality, are not yet satisfactory and require further effort; in particular, the low phase velocities of the turbulence compared with experiment needs to be addressed.

The experimental data on the spatio-temporal GAM structure from different devices do not yet fit in a unified picture. Although the GAM frequency and trends are well described by analytical models, the observation of phase locked global GAM modes over a larger radial region are puzzling. Gyrofluid simulations, however, seem to reproduce this effect.

Experimental studies on the turbulence structure confirm expected effects such as a poloidal asymmetry in the fluctuation amplitude and an increase in structure size with ion mass in the plasma core.

These first results are encouraging to pursue the project of an experimental examination of turbulence simulations carried out on well diagnosed discharges to enable comprehensive comparisons of the turbulent fluctuations including zonal flow and GAM activities from the core to the edge of plasmas at different parameters.

Acknowledgements: This project has received funding from the HGF Virtual Institute on Virtual Institute on Plasma Dynamical Processes and Turbulence Studies using Advanced Microwave Diagnostics and from the European Union's Horizon 2020 research and innovation programme under grant agreement number 633053. The views and opinions expressed herein do not necessarily reflect those of the European Commission. The support from EFDA under fusion researcher fellowship WP12-FRFIPP/Happel is gratefully acknowledged.

\section{References}

[1] P. Rohmann et al., IEEE International Symposium on Phased Array Systems \& Technology (Waltham, MA, USA, 2013), p. 559. 
[2] C. Koenen et al., German Microwave Conference (GeMiC) (Nürnberg, Germany, 2015).

[3] G. D. Conway et al., Plasma Phys. Controll. Fusion 46, 951 (2004).

[4] G. D. Conway et al., EX/P5-38, 22 ${ }^{\text {nd }}$ IAEA Fusion Energy Conference, 2008.

[5] T. Happel et al., Proc. $11^{\text {th }}$ International Reflectometry Workshop, Palaiseau, France, 2013.

[6] P. Hennequin et al., Proc. 11 ${ }^{\text {th }}$ International Reflectometry Workshop, Palaiseau, France, 2013.

[7] F. Clairet et al., Rev. Sci. Instrum. 81, 10D903 (2010).

[8] A. Krämer-Flecken et al., Nucl. Fusion 46, S730 (2006).

[9] A. Krämer-Flecken and S. Soldatov, Proc. $10^{\text {th }}$ International Reflectometry Workshop, Padua, Italy, 2011.

[10] P. Varela et al., Nucl. Fusion 46, S693 (2006).

[11] C. Lechte et al., Doppler Reflectometry Simulations for ASDEX Upgrade, Proc. $41^{s t}$ EPS Conference on Plasma Physics (Berlin, Germany, 2014).

[12] C. Tröster, Development of a flexibleDoppler reflectometry system and its application to turbulence charactrization in the ASDEX Upgrade tokamak, 2008, PhD thesis, LMU Munich, IPP Garching.

[13] G. D. Conway et al., Plasma Phys. Controll. Fusion 50, 124026 (2008).

[14] F. Jenko, W. Dorland, M. Kotschenreuther, and B. N. Rogers, Phys. Plasmas 7, 1904 (2000).

[15] C. Lechte, IEEE Transactions on Plasma Science 37, 6 (2009).

[16] E. Blanco and T. Estrada, Plasma Phys. Controll. Fusion 50, 095011 (2008).

[17] E. Z. Gusakov, A. V. Surkov, and A. Y. Popov, Plasma Phys. Controll. Fusion 47, 959 (2005).

[18] L. Vermare et al., Phys. Plasmas 18, 012306 (2011).

[19] A. Casati et al., Phys. Rev. Lett. 102, 165005 (2009).

[20] O. Gürcan et al., Phys. Rev. Lett. 102, 255002 (2009).

[21] P. Hennequin et al., Plasma Phys. Controll. Fusion 46, 121 (2004).

[22] L. Schmitz et al., Nucl. Fusion 52, 023003 (2012).

[23] G. D. Conway et al., Plasma Phys. Controll. Fusion 44, 451 (2002).

[24] A. E. White et al., Phys. Plasmas 17, (2010).

[25] M. W. Shafer et al., Phys. Plasmas 19, (2012).

[26] J. C. Hillesheim et al., Rev. Sci. Instrum. 83, (2012).

[27] R. M. McDermott et al., Plasma Phys. Controll. Fusion 53, 124013 (2011).

[28] R. M. McDermott et al., Proc. 24th Int. Conf. on Fusion Energy [EX/2-1] (San Diego, USA, 2012).

[29] M. Kotschenreuther, G. Rewoldt, and W. Tang, Comp. Phys. Comm. 88, 128 (1995).

[30] E. Fable, C. Angioni, and O. Sauter, Plasma Phys. Controll. Fusion 52, 015007 (2010).

[31] C. Angioni et al., Nucl. Fusion 51, 023006 (2011).

[32] C. Angioni et al., Plasma Phys. Controll. Fusion 51, 124017 (2009). 
[33] F. Merz and F. Jenko, Nucl. Fusion 50, 054005 (2010).

[34] T. Happel et al., Phys. Plasmas 22, 032503 (2015).

[35] A. Bañón Navarro et al., Gyrokinetic studies of core turbulence features in ASDEX Upgrade H-mode plasmas, submitted to Phys. Plasmas, http://arxiv.org/abs/1501.05163.

[36] M. A. Beer and G. W. Hammett, Phys. Plasmas 3, 4018 (1996).

[37] P. Malinov and F. Zonca, J. Plasma Phys. 71, 301 (2005).

[38] L. Vermare et al., 38th European Physical Society Conference on Plasma Physics (Strasbourg, France, 2011).

[39] Y. Camenen et al., Nucl. Fusion 47, 510 (2007).

[40] P. Sauter et al., Phys. Plasmas 21, 055906 (2014).

[41] A. Marinoni et al., Nucl. Fusion 51, 055016 (2009).

[42] L. Porte et al., Multi-Diagnostic Study of Core Turbulence and Geodesic Acoustic Modes in the TCV Tokamak, 25th IAEA Fusion Energy Conf., [EX/P3-57] (Saint Petersburg, Russia, 2014).

[43] P. Simon et al., Proc. 12 ${ }^{\text {th }}$ International Reflectometry Workshop, Jülich, Germany, 2015.

[44] Z. Gao, Plasma Sci. Technol. 13, 15 (2011).

[45] P. Simon, 2015, PhD thesis, University Stuttgart.

[46] A. Storelli, Étude du transport turbulent dans les plasmas du tokamak Tore Supra: observation des écoulements perpendiculaires stationnaires et du mode acoustique géodésique, 2015, PhD thesis, Université Politechnique, Palesau, France.

[47] J. R. Robinson et al., Phys. Plasmas 20, 052302 (2013).

[48] B. Scott, Contr. Plasma Phys. 47, 217 (2006).

[49] E. Viezzer et al., Nucl. Fusion 53, (2013).

[50] C. A. de Meijere et al., Plasma Phys. Controll. Fusion 56, 072001 (2014).

[51] G. Wang et al., Phys. Plasmas 20, 92501 (2013).

[52] T. Ido et al., Plasma Phys. Controll. Fusion 48, S41 (2006).

[53] L. Vermare et al., Nucl. Fusion 52, 063008 (2012).

[54] V. Grandgirard et al., Comm. Nonlinear Sc. and Numerical Simulation 13, 13 (2008).

[55] F. Zonca and L. Chen, Euro Phys. Letters 83, 35001 (2008).

[56] Z. Qiu, L. Chen, and F. Zonca, Phys. Plasmas 21, 022304 (2014).

[57] Y. Xu et al., Phys. Rev. Lett. 110, 265005 (2013).

[58] P. Hennequin et al., invited talk on EPS 2015, to be published in Plasma Phys. Contr. Fusion.

[59] G. Birkenmeier et al., Plasma Phys. Controll. Fusion 56, 075019 (2014).

[60] T. T. Ribeiro and B. D. Scott, Plasma Phys. Controll. Fusion 50, 055007 (2008). 